\title{
SECURE APPROACH TO SHARE DATA AMONG MULTIPLE USERS USING SINGLE-KEY TECHNIQUE
}

\begin{tabular}{|l|l|}
\hline Shwetha.M & Prof.K.S.Sampada \\
Department of Computer Science & Department of Computer Science \\
RNS Institute of Technology & RNS Institute of Technology \\
Banglore, India & Banglore, India \\
shwegowda22@gmail.com & k.s.sampada@gmail.com \\
\hline
\end{tabular}

\begin{abstract}
As Data sharing is important functionality it is necessary to securely, efficiently and flexibly share data with others, hence data owners are motivated to outsource their data for greater flexibility but to preserve data privacy these data are encrypted before outsourcing. Existing method uses public key cryptosystem where each file will be having a pair of: public and private keys. As the number of user increases, then each file will be having two keys which will lead to a problem for storing and maintaining the keys. This leads to increase in complexity. To overcome the above said problem this paper proposes a new public key cryptosystem which use key aggregation method that requires efficient delegation of decryption rights for encrypted files. This method is to aggregate any set of secret keys and make them as single key which includes the power of all the aggregated keys. The secret key holder can release a constant size aggregate key for the requested encrypted files where other encrypted files remain confidential. This aggregate key can be sent to others easily and also can be stored in smart card.
\end{abstract}

Keywords-Data sharing, Key-aggregate system, Data outsourcing, Data privacy

\section{INTRODUCTION}

The limitations in the organizations for managing and storing data and also resource constraints like storage capacity or necessity of back up of the information in mobile phones led to rise in demand for data outsourcing [1]. It is also can be used as technology behind many online services for example personal applications. Photos, emails, documents can be outsourced and can access those information by any device, from any place using wireless technology.

Data privacy is an important aspect of data sharing. With the advancement of recent technologies there is to personal information. Data privacy may be applied in numerous ways, including encryption, authentication and data masking - each attempting to ensure that information is available only to those with authorized access. These protective measures are geared toward preventing data mining and the unauthorized use of personal information, which are illegal in many parts of the world. Data privacy relates to different data types, including:

Internet privacy (online privacy): All personal data shared over the Internet is subject to privacy issues. Most websites publish a privacy policy that details the website's intended use of collected online and/or offline collected data.

Financial privacy: Financial information is particularly

sensitive, as it may easily use to commit online and/or offline fraud.

Medical privacy: All medical records are subject to stringent laws that address user access privileges. By law, security and authentication systems are often required for individuals that process and store medical records.

As data sharing is important functionality [2]. For example: if one person wants to share only some of his private documents to his friends. Considering data privacy all the documents are encrypted. The challenging task is to share the encrypted data securely among other users. Taking an illustration of Dropbox. Assume that Mary uploads some of her files into Dropbox and it does not want to leak to anyone hence she encrypt all her files using her own keys and uploads it, If Mary friend asks for some files to share, the possible ways of sharing encrypted files to her friend are:-

- Mary encrypts all files with single encryption key and gives her friend the corresponding secret key directly. It is not desirable since the data which has to remain confidential can be accessed. 
- Mary encrypts files with distinct keys and sends her friend the corresponding secret keys. Here as the number of files increases, the keys grow exponentially hence transferring and storing keys is expensive.

To overcome the issues in previous methods. In this paper we are proposing a method called key aggregate cryptosystem where data owner can encrypt a file under public key and also he holds a master secret key which is used to extract secret keys and extracted keys can be made as an aggregate key which is compact as single key so that it can be sent securely to others and can also be stored easily.

With this solution, Mary can send her friend a single key via email, her friend can download the encrypted files from Mary's Dropbox and can use this aggregate key to decrypt these encrypted files.

\section{LITERATURE SURVEY}

It provides background information on the issues to be considered and to emphasize the relevance of the present study.

Access control in a hierarchy is the scheme [3] based on cryptography is proposed for access control in a system where hierarchy is represented in tree structure. In this method, users of a computer are divided into number of disjoint sets and one user is assigned as root node and it has higher security clearance, where it can access the information held by other users but not opposite. The access control problem arises in organization where hierarchical structure exists. This approach does not completely solve the more general multilevel security problem and granting the parent key implicitly grants all the keys of its descendant nodes.

Sandhu [4] proposed a cryptographic implementation method for access control in a situation where users and information items are classified into security classes organized as a root tree, with the most privileged security class as root. Each user stores a single key of fixed size corresponding to the user's security class. Keys for security classes in the sub tree below the user's security class are generated from this key by iterative application of one-way functions. New security classes can be defined without altering existing keys. This scheme is based on conventional cryptosystems.

More advanced cryptographic key assignment schemes [5] are hierarchical access control in secure group communications where many group communications require a security infrastructure that ensures multiple levels of access privilege for group members. Access control in hierarchy is prevalent to multimedia applications which consist of users that subscribe to different quality levels or different set of data streams.

In general, hierarchical approaches can solve the problem partially if one intends to share all files under a certain branch in the hierarchy and also number of keys increases with number of branches.

Identity based encryption [6] is a public key system where arbitrary string such as email address can be used as public key and secret key is issued from private key generator which contains master secret key with respect to user identity. The encryption of message can be done by taking public parameter and user identity and also decryption can be done by secret key. New type of identity based encryption is called as fuzzy identity-based encryption [7] which views an identity as set of descriptive attributes. This method allows for a private key for an identity ' $a$ ' to decrypt a ciphertext encrypted with an identity a', if and only if the identities a and a' are close to each other. The advantage of fuzzy IBE is document can be stored on a simple untrusted storage server instead of relying on trusted server to perform authentication checks before delivering a document. However in fuzzy IBE, one single compact secret key can decrypt ciphertexts encrypted under many identities which are close in certain metric space but not for an arbitrary set of identities and therefore it does not match with idea of key aggregation.

Hierarchical identity based encryption [8] is a generalization of identity based encryption that represents an organizational hierarchy. An identity at level $\mathrm{k}$ of the hierarchy tree can issue private key to its descendant identities but cannot 
decrypt message. It allows a root public key generator to distribute the workload by delegating public key generation and identity authentication to lower level public key generators.

In attribute based encryption [9], a user's keys and ciphertexts are labelled with set of descriptive attributes and a particular key can decrypt a particular ciphertext only if there is a match between the attributes of the ciphertext and the user's key and it allows for decryption only when at least $\mathrm{k}$ attributes overlapped between a ciphertext and private key. The main problem in this method is compactness of secret keys, because the size of key increases as the number of attributes increases.

Hierarchical attribute set based encryption [10] is represented by hierarchical structure of users. HASBE employs multiple value assignments for access expiration time to deal with user revocation more efficiently than existing schemes. The drawback in this scheme is that it applies cryptographic methods by disclosing data decryption keys only to authorize users. These solutions introduce a heavy computation overhead on the data owner for key distribution and data management when fine grained data access control is desired, thus do not scale well.

Proxy re-encryption [11] is the scheme in which ciphertext can be decrypted without sending the secret key to the delegate. In proxy re-encryption scheme, a proxy is given special information that allows it to translate a ciphertext under one key into ciphertext of the same message under a different key. This leads to separate interaction with the proxy for the decryption each time which is inconvenient.

Benaloh et.al [12] proposed an encryption scheme which is proposed for concisely transmitting large number of keys in broadcast scenario. The key derivation process for a set of classes is as follows: A composite modulus is chosen where $\mathrm{p}$ and $\mathrm{q}$ are two large random primes. Master secret key is chosen randomly. A constant size key for set can be generated and these key can be given for those have been delegated the access rights for $\mathrm{S}$. but it is designed for symmetric-key where the content provider needs to get the corresponding secret keys to encrypt data which is not suitable for many applications.

\section{PROPOSED SYSTEM}

In this paper we propose key aggregate cryptosystem framework where data owner establishes the account and generates the public/master secret key, messages can be encrypted from anyone who decide to produce ciphertext, aggregation key for set of files can be extracted from master-secret key, generated key can be send to other user securely and can decrypt the message. We consider three modules for the frame work.

\section{A. Data owner Module}

In data owner module, the owner can perform operations like selecting of files, key generation, encryption and uploading of files. Data owner setup an account on an untrusted server, selects the file which as to be encrypted, for each selected file key is generated (public and mastersecret key pair), the selected file is encrypted under public key and it is uploaded to the server.

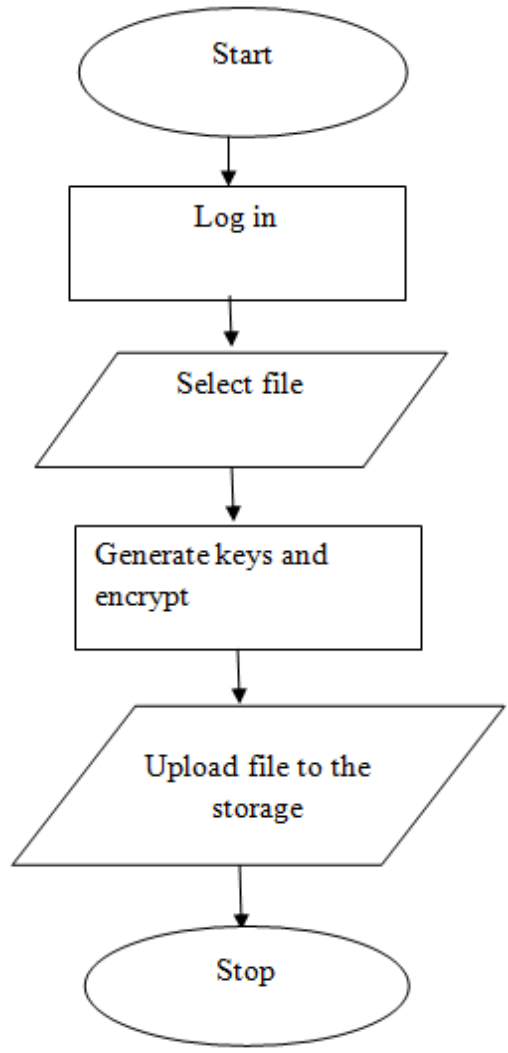

Fig.1. flow chart for data owner 


\section{B.Key aggregation Module}

In this module, data user downloads the encrypted files which is required from server, since the file is encrypted and he could not access the information, the data user requests the secret key to the data owner for decrypting the selected encrypted files, the data owner extracts the master secret key of each file and aggregates them into single key and send to the user.

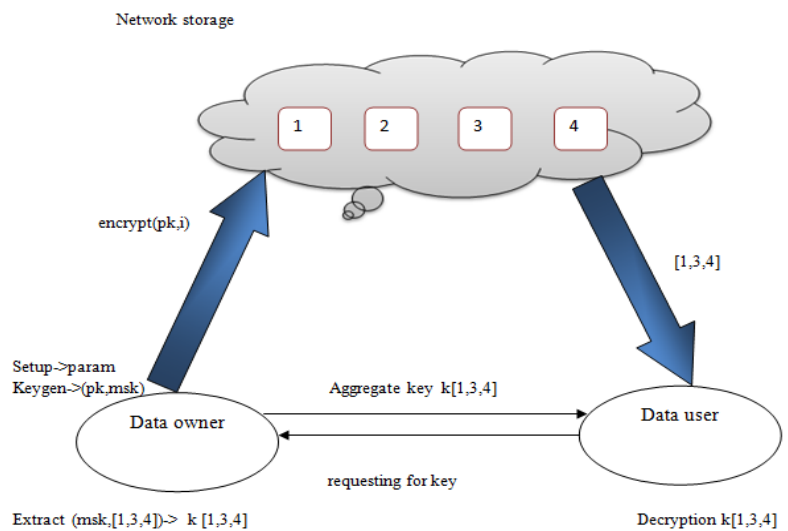

Fig 2: secure data sharing using single key technique

\section{C.Decryption Module}

When the data user receives an aggregate key, by applying aggregate key to the selected encrypted files it can be decrypted and original file can be produced.

The main application of key aggregate cryptosystem is data sharing, it enables to share data in confidential and selective way by distributing to each authorized users a single and small aggregate key.

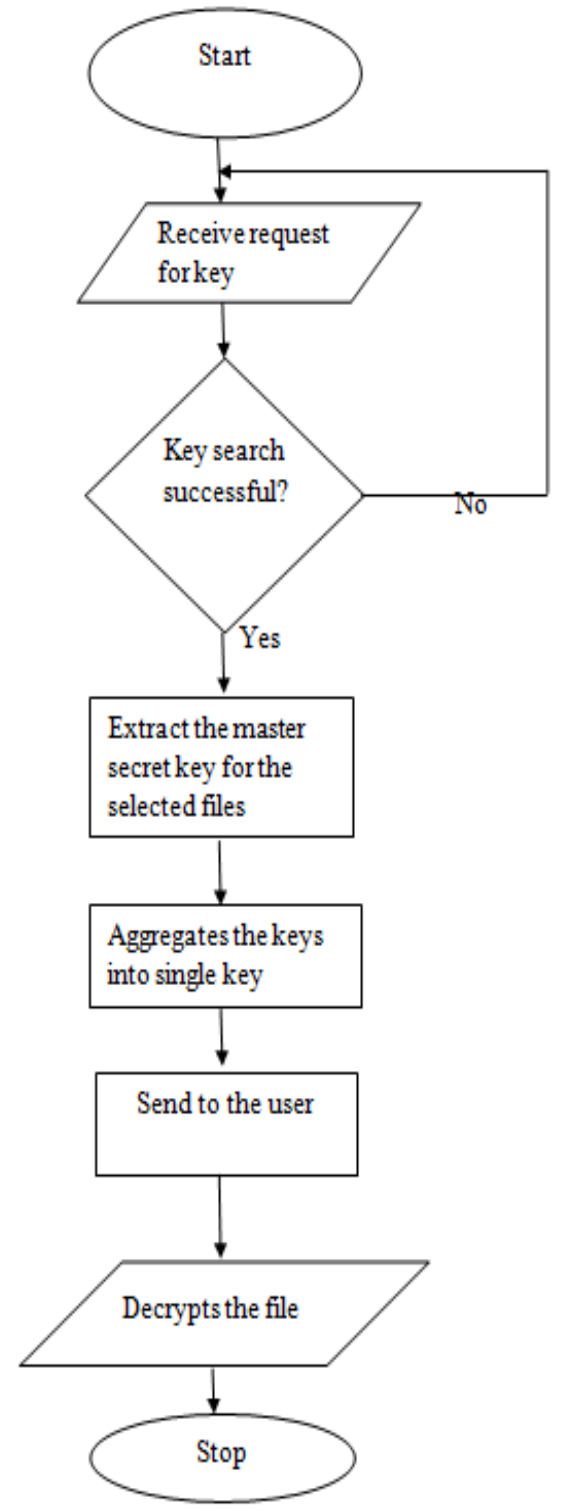

Fig.3. flow chart for key aggregation

\section{ALGORITHM FOR KEY AGGREGATE CRYPTOSYSTEM}

The key-aggregate encryption [13] as five steps as follows:

- Set up phase : Randomly pick bilinear group $G$ of prime order $p$ where $G$ is bilinear group if $\mathrm{G}$ and $\mathrm{GT}$ be two cyclic groups of prime order $p$ and $\hat{e}: \mathbb{G} \times \mathbb{G} \rightarrow \mathbb{G}_{T}$ is a bilinear map with the following properties:-

1) Bilinear: - for all $\mathrm{u}, \mathrm{v} \in \mathrm{G}$ and a, $\mathrm{b} \in \mathrm{Z}$, we have e (ua, vb) $=\mathrm{e}(\mathrm{u}$, v) ab. 
2) Non -degenerate:- $\mathrm{e}(\mathrm{g}, \mathrm{g}) \neq 1$, a generator $\mathrm{g} \in \mathrm{G}$ where generator is an element where repeated application of generator on themselves produces back the elements in the group and $\alpha$ that belongs to the non zero prime numbers. Compute $g_{i}=g^{\alpha^{*}} \in \mathbb{G}^{\mathrm{H}}$ for each file0. Output the system parameter as param $=$ $\left\langle g, g_{1}, \ldots, g_{n}, g_{n+2}, \ldots, g_{2 n}\right\rangle$.

- Key generation phase: pick $\gamma$ from non zero prime numbers, the public key is $\mathrm{pk}=$ $g^{\gamma}$ and master secret key is $\mathrm{msk}=\gamma$.

- Encryption phase: select any message $\mathrm{m}$ that belongs to the group GT and the index of the message i.e. number of file, randomly pick $t$ from non zero prime numbers and encrypt the message ${ }_{\text {as }} \mathcal{C}=\left\langle g^{t},\left(\operatorname{vg}_{i}\right)^{t}, m \cdot \hat{e}\left(g_{1}, g_{n}\right)^{t}\right\rangle$.

- Extract phase: The aggregate key is computed by extracting the master secret keys of set of files as

$\mathrm{Ks}=\prod_{j \in \mathcal{S}} g_{n+1-j}^{\gamma}$.

- Decryption phase: The aggregate key is applied to set of encrypted files that has been downloaded and decryption of the message is done which produces back the original message as: $m=c_{3} \cdot \hat{e}\left(K_{\mathcal{S}}\right.$.

$$
\left.\prod_{j \in \mathcal{S}, j \neq i} g_{n+} 1-j+i, c_{1}\right) / \hat{e}\left(\prod_{j \in \mathcal{S}} g_{n+1-j}, c_{2}\right)
$$

\section{CONCLUSION}

The main concept is to provide data privacy. This paper focuses on compressing of secret keys into a single compact key which has the power of all aggregated keys. The only files which are selected can be decrypted but where the other files remain confidential. The aggregated key can be easily sent to the others and maintained easily.

\section{REFERENCES}

[1] C. Wang, S.S.M. Chow, Q. Wang, K. Ren, and W. Lou, "Privacy-

Preserving Public Auditing for Secure Cloud Storage," IEEE Trans.

Computers, vol. 62, no. 2, pp. 362-375, Feb. 2013.

[2] B. Wang, S.S.M. Chow, M. Li, and H. Li, "Storing Shared Data on

the Cloud via Security-Mediator," Proc. IEEE 33rd Int'l Conf.

Distributed Computing Systems (ICDCS), 2013.

[3] G. Ateniese, A.D. Santis, A.L. Ferrara, and B. Masucci, "Provably-

Secure Time-Bound Hierarchical Key Assignment Schemes,"

J. Cryptology, vol. 25, no. 2, pp. 243-270, 2012.

[4] R.S. Sandhu, "Cryptographic Implementation of a Tree Hierarchy

for Access Control," Information Processing Letters, vol. 27, no. 2, pp. 95-98, 1988.

[5] M.J. Atallah, M. Blanton, N. Fazio, and K.B. Frikken, "Dynamic and Efficient Key Management for Access Hierarchies," ACM

Trans. Information and System Security, vol. 12 , no. 3 , pp. 18:1-

18:43,2009.

[6] D. Boneh and M.K. Franklin, "IdentityBased Encryption from the

Weil Pairing," Proc. Advances in Cryptology (CRYPTO '01), vol. 2139, pp. 213-229, 2001.

[7] A. Sahai and B. Waters, "Fuzzy IdentityBased Encryption," Proc.

22nd Int'l Conf. Theory and Applications of Cryptographic

Techniques (EUROCRYPT '05), vol. 3494, pp. 457-473, 2005.

[8] Adi Shamir. Identity-based cryptosystems and signature schemes. In

Proceedings of CRYPTO 84 on Advances in cryptology pages $47-53$. 
Springer-Verlag New York, Inc., 1985.

[9] V. Goyal, O. Pandey, A. Sahai, and B.

Waters, "Attribute-Based

Encryption for Fine-Grained Access Control of Encrypted Data,"

Proc. 13th ACM Conf. Computer and Comm. Security (CCS '06),

pp. 89-98, 2006.

[10] Zhiguo Wan, Jun'e Liu, and Robert H.

Deng, Senior Member, IEEE,

"HASBE: A Hierarchical Attribute-Based

Solution for Flexible and

Scalable Access Control in Cloud Computing"in Proc.IEE Transactions

on Information Forensics and Security, vol.7, No.2, April 2012.

[11] C.-K. Chu and W.-G. Tzeng, "Identity-

Based Proxy Re-encryption

without Random Oracles," Proc.

Information Security Conf.

(ISC '07), vol. 4779, pp. 189-202, 2007.

[12] J. Benaloh, M. Chase, E. Horvitz, and K.

Lauter, "Patient Controlled

Encryption: Ensuring Privacy of Electronic Medical Records," Proc.

ACM Workshop Cloud Computing Security

(CCSW '09), pp. 103-

114, 2009.

[13] D. Boneh, C. Gentry, and B. Waters,

"Collusion Resistant Broadcast

Encryption with Short Ciphertexts and

Private Keys," Proc.

Advances in Cryptology Conf. (CRYPTO

'05), vol. 3621, pp. 258

275, 2005. 